\title{
Improved clinical outcomes of preimplantation genetic testing for aneuploidy using MALBAC-NGS compared with MDA-SNP array
}

Wenbin Niu ${ }^{1,2+}$, Linlin Wang ${ }^{1,2+}$, Jiawei $\mathrm{Xu}^{1,2}$, Ying $\mathrm{Li}^{1,2}$, Hao Shi ${ }^{1,2}$, Gang Li ${ }^{1,2}$, Haixia Jin ${ }^{1,2}$, Wenyan Song ${ }^{1,2}$, Fang Wang ${ }^{1,2}$ and Yingpu Sun ${ }^{1,2^{*}}$

\begin{abstract}
Background: To assess whether preimplantation genetic testing for aneuploidy with next generation sequencing (NGS) outweighs single nucleotide polymorphism (SNP) array in improving clinical outcomes.

Methods: A retrospective analysis of the clinical outcomes of patients who underwent PGT-A treatment in a single center from January 2013 to December 2017.A total of 1418 couples who underwent PGT-A treatment were enrolled, of which 805 couples used NGS for PGT-A, while the remaining 613 couples used SNP array for PGT-A. Clinical pregnancy rate, miscarriage rate and healthy baby rate were compared between the MALBAC-NGS-PGT-A and MDA-SNP-PGT-A groups.

Results: After testing karyotypes of 5771 biopsied blastocysts, 32.2\% (1861/5771) were identified as chromosomally normal, while $67.8 \%$ were chromosomally abnormal. In terms of clinical outcomes, women in the MALBAC-NGSPGT-A group had a significantly higher clinical pregnancy rate $(50.5 \%$ vs $41.7 \%, p=0.002)$ and healthy baby rate (39.6\% vs $31.4 \%, p=0.003)$, and a lower miscarriage rate (15.5\% vs $22.8 \%, p=0.036)$.
\end{abstract}

Conclusion: This is the largest study reporting the extensive application of NGS-based PGT-A, whilst comparing the clinical outcomes of MALBAC-NGS-PGT-A and MDA-SNP-PGT-A. The results provide greater evidence supporting the wider use of NGS in PGT-A, not only for its lower cost but also for its improved clinical outcomes compared to SNP-based PGT-A.

Keywords: Preimplantation genetic testing for aneuploidy, Single nucleotide polymorphism, Next generation sequencing, Pregnancy outcome

\footnotetext{
* Correspondence: syp2008@vip.sina.com

${ }^{+}$Wenbin Niu and Linlin Wang contributed to the work equally and should be regarded as co-first authors.

${ }^{1}$ Center for Reproductive Medicine, The First Affiliated Hospital of Zhengzhou

University, Eastern Jianshe Road, Erqi District, Zhengzhou City, Henan

Province, People's Republic of China

${ }^{2}$ Henan Key Laboratory of Reproduction and Genetics, The First Affiliated Hospital of Zhengzhou University, Eastern Jianshe Road, Erqi District,

Zhengzhou City, Henan Province, People's Republic of China
}

(C) The Author(s). 2020 Open Access This article is licensed under a Creative Commons Attribution 4.0 International License, which permits use, sharing, adaptation, distribution and reproduction in any medium or format, as long as you give appropriate credit to the original author(s) and the source, provide a link to the Creative Commons licence, and indicate if changes were made. The images or other third party material in this article are included in the article's Creative Commons licence, unless indicated otherwise in a credit line to the material. If material is not included in the article's Creative Commons licence and your intended use is not permitted by statutory regulation or exceeds the permitted use, you will need to obtain permission directly from the copyright holder. To view a copy of this licence, visit http://creativecommons.org/licenses/by/4.0/ The Creative Commons Public Domain Dedication waiver (http://creativecommons.org/publicdomain/zero/1.0/) applies to the data made available in this article, unless otherwise stated in a credit line to the data. 


\section{Background}

Estimates have indicated that only one-third of natural conceptions progress to a healthy live birth [1,2]. Even after far advanced assisted reproductive technology (ART) was introduced for the management of many kinds of subfertility, the healthy baby rate for embryo transfer cycles was still reported to be less than 30\% [3]. Fetal chromosomal aberrations have been recognized as the leading cause of the poor healthy live birth rate, and account for up to $70 \%$ of miscarriages [4]. Additionally, various chromosomal abnormalities can be detected in both younger and older women [4]. A study even found that more than $90 \%$ of blastomeres in human preimplantation embryos had at least one chromosomal abnormality in one or more cells [5]. Therefore, in theory, transferring chromosomally normal embryos should be an effective way to reduce the miscarriage rate as well as to increase the pregnancy rate.

Preimplantation genetic testing for aneuploidy (PGTA) has been used for decades to minimize chromosomally abnormal pregnancies and to select chromosomally normal embryos prior to transfer, which is reported to have remarkably reduced the risk of an affected pregnancy and miscarriage $[3,6,7]$. This treatment benefits couples with chromosome abnormalities, repeated IVF failures (occurring more than three times) and those with advanced maternal age (over 35 years) [8].

Fluorescence in situ hybridization (FISH) was the first molecular cytogenetic technique widely used in PGT-A treatment but the limited number of available fluorescent probes targeted at whole chromosomes, and complex sample preparation procedures, restricted its application [9]. In contrast, array-based genome-wide techniques such as single nucleotide polymorphism (SNP) microarray allow every chromosome to be evaluated simultaneously [10]. Studies have shown that arraybased PGT-A treatment may increase the pregnancy rate to $69.4 \%$ compared to FISH which has a pregnancy rate of $38.4 \%$ [11]. However, its relatively high cost may restrict its usage in clinical practice.

Recent advances in NGS are increasing its range of application in PGT-A clinical practice. The accuracy of NGS in detecting chromosomally abnormal embryos has been extensively reported [12-14]. Both NGS and SNP array rely on whole genome amplification (WGA) for a single-cell biopsy to generate enough DNA. The most common WGA methods are multiple displacement amplification (MDA) for SNP array and multiple annealing and looping-based amplification cycles (MALBAC) for NGS. Studies have suggested that MDA and MALBAC possess similar single-nucleotide variant detection, false-positive rates and allelic dropout rates [15].

However, very few studies have reported the clinical outcomes of NGS-based PGT-A treatment, therefore this study compares clinical outcomes between NGSbased and SNP array-based PGT-A, including the clinical pregnancy rate, miscarriage rate and healthy baby rate, to contribute to this field of research.

\section{Methods \\ Patients}

This study was approved by the Ethics Committee of The First Affiliated Hospital of Zhengzhou University. Between January 2013 and December 2017, a total of 1418 couples received PGT-A treatment after being evaluated by both a geneticist and an infertility specialist in the reproductive center of The First Affiliated Hospital of Zhengzhou University. Factors leading to PGT-A treatment included abnormal karyotypes, advanced maternal age (AMA $\geq 35$ years), repetitive implantation failures (RIF $\geq 3$ times) and/or recurrent miscarriage ( $R M \geq$ 2times). To avoid DNA contamination from the paternal genome, all PGT-A patients underwent intracytoplasmic sperm injection (ICSI) treatment irrespective of their sperm quality.

The patients were divided into two main groups: MALBAC-NGS-PGT-A group and MDA-SNP-PGT-A group. Each group was subsequently classified into five subgroups according to the treatment indications of couples: Robertsonian translocation, reciprocal translocation, inversion, sex chromosome abnormality karyotype and undergoing PGS (patients with AMA or RIF or RM) treatment.

In the MALBAC-NGS-PGT-A group, a total of 805 couples were treated between January 2016 and December 2017: 157 with a Robertsonian translocation, 405 with a reciprocal translocation, 40 with an inversion, 92 with a sex chromosome abnormality karyotype and 111 with PGS treatment indications. Among the couples undergoing PGS treatment,54 were of advanced maternal age, 47 had experienced recurrent miscarriages and 10 were patients with repeated implantation failures.

In the MDA-SNP-PGT-A group, 613 couples were treated from January 2013 to December 2015. Five hundred nineteen couples were found to be chromosomally abnormal. Among them, the number of couples with a Robertsonian translocation, reciprocal translocation, inversion and sex chromosome abnormality karyotype were 105, 342, 14 and 58 respectively. The remaining 94 couples underwent PGS treatment due to advanced maternal age (39 couples), recurrent miscarriages (46 couples) and repeated implantation failures (9 couples).

\section{Controlled ovarian stimulation, embryo biopsy and transfer}

Controlled ovarian stimulation (COS) was performed using the standard long protocol. Gonadotropinreleasing hormone agonist $(\mathrm{GnRH}-\mathrm{a})$, Decapeptyl 
(Germany) or Diphereline (France) were used to induce complete down-regulation (follicle stimulating hormone $\mathrm{FSH} \leq 5 \mathrm{IU} / \mathrm{L}$, luteinizing hormone $\mathrm{LH} \leq 5 \mathrm{IU} / \mathrm{L}$, estrogen $\mathrm{E} 2 \leq 50 \mathrm{pg} / \mathrm{ml}$, diameter of the largest follicle $\leq 10 \mathrm{~mm}$ and endometrial thickness $\leq 5 \mathrm{~mm}$ ). Gonal-F (Gn, Switzerland) was subsequently administered. The starting dose of $\mathrm{Gn}$ was determined based on body mass index, age, basal FSH level and antral follicle count. Where the diameter of the dominant follicle was more than $14 \mathrm{~mm}$, fasting blood was collected every day to determine the levels of LH, E2 and P present. The regimen of $\mathrm{Gn}$ was adjusted based on the hormone levels and follicle size. When the amount of follicles with a diameter $\geq$ $16 \mathrm{~mm}$ was more than two-thirds, and the diameter of the dominant follicle was greater than $20 \mathrm{~mm}$, human chorionic gonadotropin was intramuscularly injected. Thirty-seven hours later, oocyte retrieval was performed using ultrasonic guidance. Fertilization took place $3-4 \mathrm{~h}$ after oocyte retrieval. Embryo cleavage was recorded every $24 \mathrm{~h}$. Blastocysts were graded on the fifth or sixth day after oocyte retrieval using the Gardner criteria. Trophectoderm (TE) biopsy was performed on selected blastocysts higher than $3 \mathrm{BB}$, and 5 to $10 \mathrm{TE}$ cells were isolated and cut using a laser. The biopsied cells were then immediately placed in the RNAse and DNAse-free polymerase chain reaction tube and transported to our PGT-A center. After testing karyotypes of the biopsied blastocysts, a single thawed euploid embryo was transferred for each transfer cycle.

\section{NGS testing and data analysis}

MALBAC was used for WGA in NGS testing, following the manufacturer's protocol (XK028,Yikon Genomics, China). Amplified DNA was then diluted, fragmented, adaptor ligated, subjected to PCR reaction and purified. After measuring the concentration, libraries were processed using rapid single-end 50 cycle mode sequencing using a Hiseq 2500 sequencer (Illumina, USA) in our center.

The sequenced raw data were demultiplexed and converted to the FASTQ format using CASAVA 1.8.4(Illumina, USA).MALBAC primers, adaptors and low-quality bases were removed from the FASTQ data using Trimmomatic [16],generating an average of $\sim 2$ million filtered reads per sample, with an average $\sim 0.03 \times$ sequencing depth.

High-quality reads were mapped to a hg19 reference genome using the Burrows-Wheeler Aligner software package [17]. Unique mapped reads were extracted from the alignment reads (bam file). The reference genome was then divided into non-overlapping observation windows (bins) with a size of $1000 \mathrm{~Kb}$. The number of reads and GC-content were calculated in each bin and GC bias correction was performed for every $1 \%$ GC-content [18].
The $\mathrm{R}$ programming language was then used to graph the final relative number of reads for each bin to visualize copy number variations.

\section{SNP array and data analysis}

MDA was performed using REPLI-g Single Cell Kit (150, 345, QIAGEN, Germany) in SNP array according to the authors' previous report [19]. Briefly, biopsied TE cells (5 to 10 cells) were lysed in DTT and DLB buffer. Whole genome DNA was amplified in an amplification buffer created by mixing REPLI-g single-cell DNA polymerase and the reaction buffer. The MDA product was then hybridized on the Illumina HumanCyto12 microarray by following the user manual. Data were analyzed using GenomeStudio Software (2011, Illumina, USA). The genotype was evaluated by using the $\mathrm{B}$ allele frequency and $\log _{2} R$ ratio $\left(\log _{2} R=\log _{2}\right.$ normalized $R$ value/expected normalized $R$ value).

\section{Clinical outcomes and statistical analysis}

Chromosomally normal/balanced blastocysts were transferred. 35 to 45 days later, clinical pregnancy was confirmed by the results of an ultrasound examination. Clinical pregnancy rate (clinical pregnancy cycles/embryo transferred cycles), miscarriage rate (embryo lost cycles/clinical pregnancy cycles) and healthy baby rate (healthy babies/ number of transferred embryos) were recorded as the main outcomes. SPSS 21.0 (IBM, USA) was used for statistical analysis. Analysis of the maternal age, BMI, basal FSH level and endometrial thickness was made using Student's t-test. Pearson's chi-squared test was also used to analyze categorical data. A $P$-value $\leq 0.05$ was considered significant.

\section{Results}

\section{Results of NGS tested blastocysts}

NGS testing was successfully performed on 805 couples and 3321 biopsied blastocysts. As is shown in Tables 1, 766 (23.1\%) euploid/normal and 2555(76.9\%)chromosomally abnormal samples were identified. Of these abnormalities, $760(22.9 \%)$ had numerical abnormalities, 404(12.2\%)contained structural abnormalities (deletions and duplications), while 1391(41.8\%) had complex abnormality (defined as two or more duplications/deletions involving the same, or two or more chromosomes) (Fig. 1).

The most common chromosome abnormality type in each subgroup was also determined, as shown in Fig. 2. Numerical abnormality was most frequently found in blastocysts from couples with Robertsonian translocation (43.8\%, 264/603), sex chromosome abnormality (27.5\%, 95/345) and PGS couples (33.2\%, 131/395), while structural abnormality showed a relatively balanced distribution among the five subgroups. Meanwhile, complex 
Table 1 NGS testing for blastocysts

\begin{tabular}{lllllll}
\hline Clinical data & \multicolumn{2}{l}{ Couples } & & & & Total \\
\cline { 2 - 5 } & $\begin{array}{l}\text { Robertsonian } \\
\text { translocation }\end{array}$ & $\begin{array}{l}\text { Reciprocal } \\
\text { translocation }\end{array}$ & Inversion & PGS & \multicolumn{2}{l}{$\begin{array}{l}\text { Sex chromosome } \\
\text { abnormality }\end{array}$} \\
\hline No. of cases & 157 & 405 & 40 & 111 & 92 & 805 \\
Maternal age (mean \pm SD) & $30.2 \pm 4.9$ & $29.4 \pm 4.09$ & $30.0 \pm 4.03$ & $35.4 \pm 5.35$ & $28.7 \pm 4.06$ & $30.3 \pm 4.76$ \\
No. of blastocysts biopsied & 603 & 1825 & 153 & 395 & 345 & 3321 \\
Euploidy & $164(27.2 \%)$ & $313(17.2 \%)$ & $54(35.3 \%)$ & $130(32.9 \%)$ & $105(30.4 \%)$ & $765(23.1 \%)$ \\
Numerical abnormality & $264(43.8 \%)$ & $241(13.2 \%)$ & $29(18.9 \%)$ & $131(33.2 \%)$ & $95(27.5 \%)$ & $760(22.9 \%)$ \\
Structural abnormality & $41(6.8 \%)$ & $237(13.0 \%)$ & $22(14.4 \%)$ & $42(10.6 \%)$ & $62(18.0 \%)$ & $404(12.2 \%)$ \\
Complex abnormality & $134(22.2 \%)$ & $1034(56.6 \%)$ & $48(31.4 \%)$ & $92(23.3 \%)$ & $83(24.1 \%)$ & $1391(41.8 \%)$ \\
\hline
\end{tabular}

abnormality was more common than the other kinds of abnormalities for couples with reciprocal translocation (56.6\%, 1034/1825) and inversion (31.4\%, 48/153) (Table 1).

\section{Results of SNP array tested blastocysts}

Among the 2450 blastocysts tested by SNP array from 613 couples, 1095 (44.7\%) were euploid/normal and 1355(55.3\%) were abnormal. Among all of the detected abnormalities, 654 (26.7\%) had a numerical abnormality, $393(16 \%)$ had a structural abnormality (duplication or deletion), 299 (12.2\%) contained complex abnormalities, and the remaining $9(0.4 \%)$ showed uniparental disomy (UPD) (Fig. 1).

With regard to the distribution of chromosomal aberration types among each subgroup, numerical abnormality was most commonly found in samples from couples with Robertsonian translocation, inversion, PGS and sex chromosome abnormality, accounting for 38.7\% (177/ $457), 32.9 \%$ (27/82), $28.3 \%(86 / 303)$ and $34.9 \%(75 / 215)$ for each of the above, respectively. Among couples with reciprocal translocation, structural abnormality (24.3\%, 338/1393) was identified to be the most frequent (Table 2 and Fig. 3).

\section{Clinical outcomes}

Among the 805 couples in the MALBAC-NGS-PGT-A group, 270 had no euploid/normal embryos to transfer, 522 underwent embryo transfer, and 13 are still waiting for transfer. Of the 522 couples with embryo transfer, 675 euploid/normal embryos were transferred in $675 \mathrm{cy}$ cles. Clinical pregnancy was identified in 341 (50.5\%) of the transferred cycles. Healthy baby rate was $39.6 \%$ (267/675,including two onset of twins). Of the clinical pregnancy cycles, $15.5 \%(54 / 341)$ of the cycles ended with miscarriage. In the SNP array-PGT-A group, 145 couples were found with no euploid embryos to transfer, while 468 couples had euploid/normal embryos. To date, 452 couples have undergone embryo transfer while 12 couples were excluded in this study because they

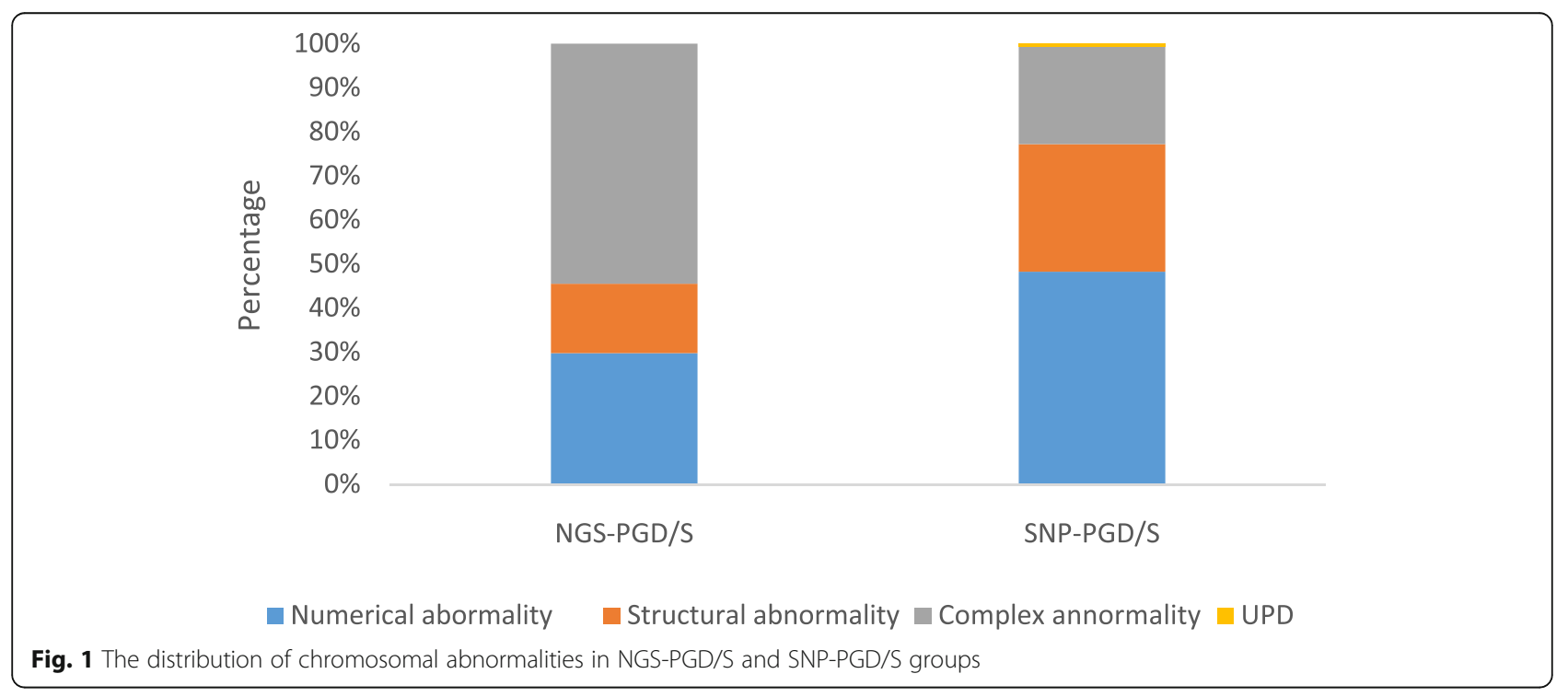




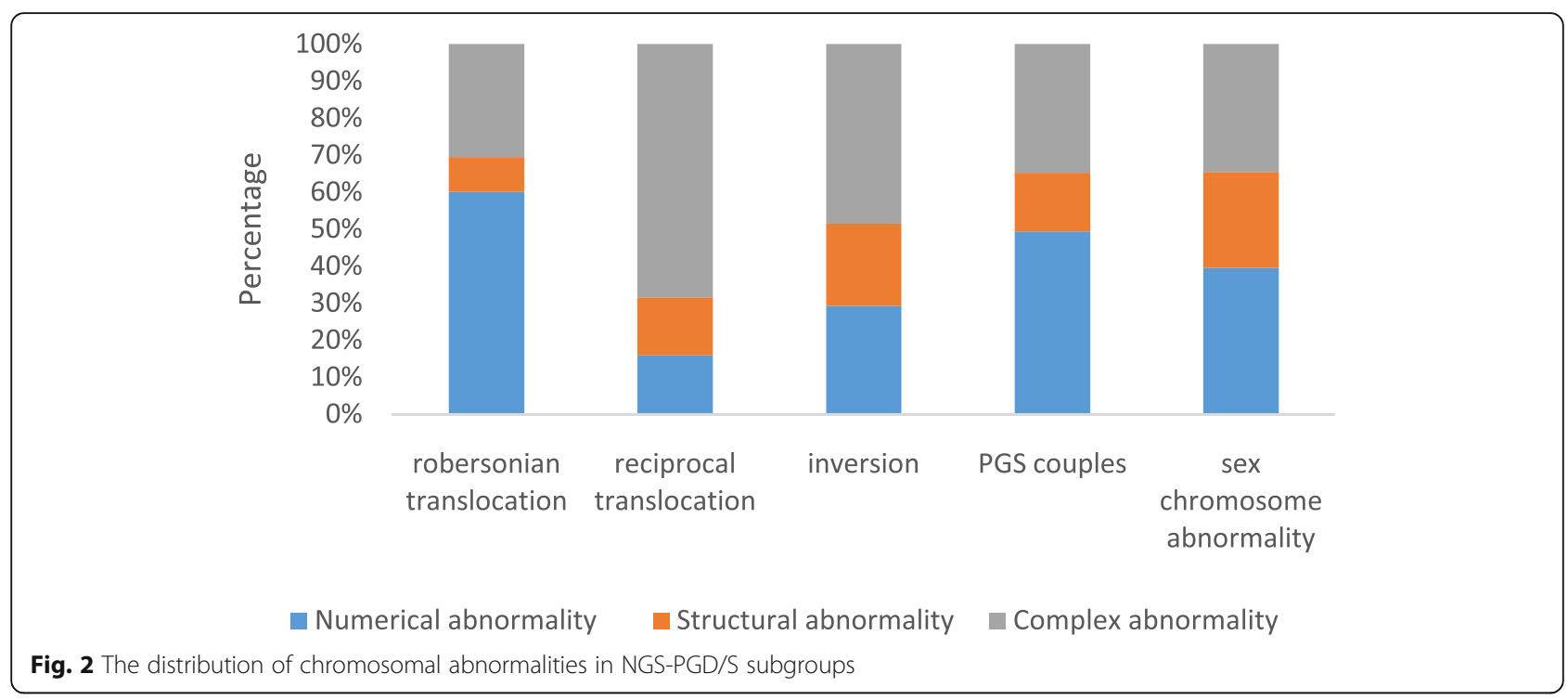

transfered two embryos in once transfer cycle, and 16 couples are still waiting for transfer. Of the 440 couples with embryo transfer, 547 euploid/normal embryos were transferred in 547 cycles. Of the 547 transferred cycles, $41.7 \%(228 / 547)$ of the cycles resulted in clinical pregnancy, healthy baby rate was $31.4 \%$ (172/547,including two onset of twins). Of the clinical pregnancy cycles, $22.8 \%(52 / 228)$ of these miscarried.

There were no significant differences in terms of maternal age, BMI, basal FSH level and endometrial thickness on the day of embryo transfer between the MALBAC-NGS-PGT-A and MDA-SNP-PGT-A groups $(P>0.05)$. However, a significantly higher clinical pregnancy rate $(50.5 \%$ vs $41.7 \%, P=0.002)$, healthy baby rate (39.6\% vs31.4\%, $P=0.003$ ) and lower miscarriage rate (15.5\% vs22.8\%, $P=0.036$ ) were observed in the MALBAC-NGS-PGT-A group when compared with the MDA-SNP-PGT-A group (Table 3).

\section{Discussion}

The study retrospectively analyzed the biopsy results of 5771 blastocysts to evaluate the clinical outcomes of NGS-based PGT-A treatment. A comparison between the clinical outcomes of women in the MALBAC-NGSPGT-A and MDA-SNP-PGT-A groups showed that the MALBAC-NGS-PGT-A group had a significantly higher clinical pregnancy rate, healthy baby rate and lower miscarriage rate, supporting the application of NGS-based PGT-A treatment to in patients with chromosomal abnormalities, advanced maternal age, repeated IVF failures and/or recurrent miscarriage.

After testing the karyotypes of a total of 5771 biopsied blastocysts, only $32.2 \%(1861 / 5771)$ of embryos were identified as chromosomally normal, and more than half of the embryos were found with various types of chromosomal abnormalities present, indicating the necessity of normal/euploid embryo selection, especially

Table 2 SNP array testing for blastocysts

\begin{tabular}{|c|c|c|c|c|c|c|}
\hline \multirow[t]{2}{*}{ Clinical data } & \multicolumn{5}{|l|}{ Couples } & \multirow[t]{2}{*}{ Total } \\
\hline & $\begin{array}{l}\text { Robertsonian } \\
\text { translocation }\end{array}$ & $\begin{array}{l}\text { Reciprocal } \\
\text { translocation }\end{array}$ & Inversion & PGS & $\begin{array}{l}\text { Sex chromosome } \\
\text { abnormality }\end{array}$ & \\
\hline No. of cases & 105 & 342 & 14 & 94 & 58 & 613 \\
\hline Maternal age $($ mean $\pm S D)$ & $29.7 \pm 4.18$ & $29.1 \pm 4.24$ & $\begin{array}{l}30.9 \pm \\
4.08\end{array}$ & $33.9 \pm 4.97$ & $29.7 \pm 4.29$ & $29.9 \pm 4.68$ \\
\hline No.of blastocysts biopsied & 457 & 1393 & 82 & 303 & 215 & 2450 \\
\hline Euploidy(\%) & $257(56.2 \%)$ & 497 (35.7\%) & $42(51.2 \%)$ & $\begin{array}{l}188 \\
(62.0 \%)\end{array}$ & $111(51.6 \%)$ & $\begin{array}{l}1095 \\
(44.7 \%)\end{array}$ \\
\hline $\begin{array}{l}\text { Numerical abnormality } \\
\text { (\%) }\end{array}$ & $177(38.7 \%)$ & $289(20.7 \%)$ & $27(32.9 \%)$ & $86(28.3 \%)$ & $75(34.9 \%)$ & $654(26.7 \%)$ \\
\hline Structural abnormality (\%) & $13(2.8 \%)$ & $338(24.3 \%)$ & $7(8.5 \%)$ & $19(6.3 \%)$ & $16(7.4 \%)$ & $393(16 \%)$ \\
\hline Complex abnormalities(\%) & $9(2.0 \%)$ & $266(19.1 \%)$ & $5(6.0 \%)$ & $10(3.3 \%)$ & $9(4.2 \%)$ & $299(12.2 \%)$ \\
\hline UPD (\%) & $1(0.2 \%)$ & $3(0.2 \%)$ & $1(1.2 \%)$ & 0 & $4(1.9 \%)$ & $9(0.4 \%)$ \\
\hline
\end{tabular}




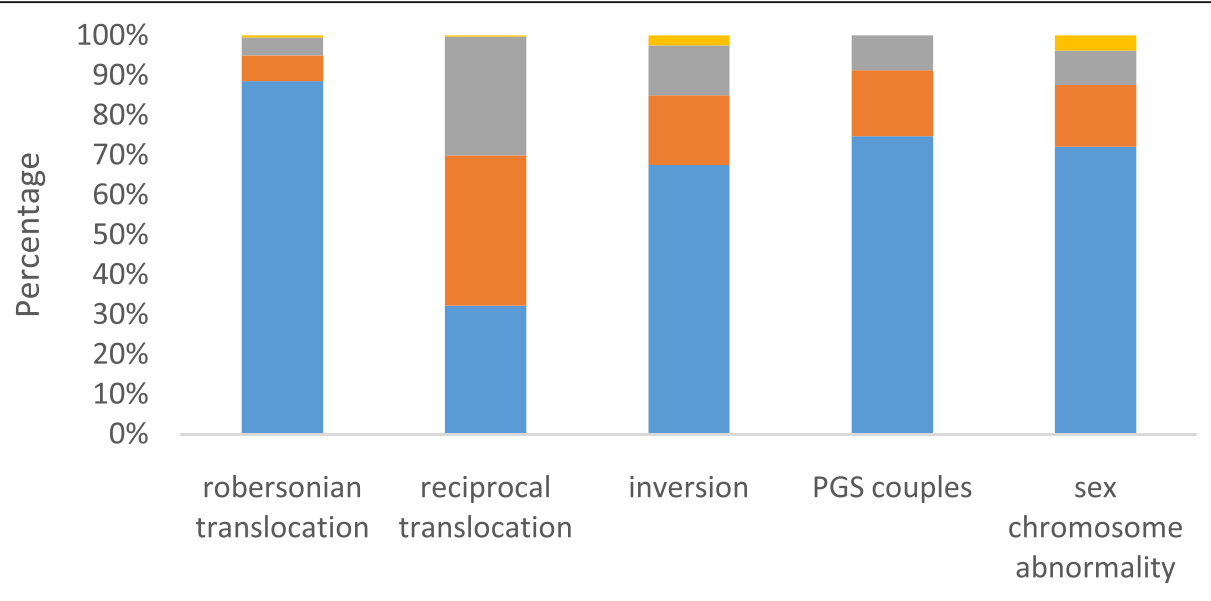

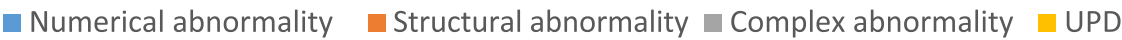

Fig. 3 The distribution of chromosomal abnormalities in SNP-PGD/S subgroups

for couples with abnormal karyotypes. Since the first successful birth using NGS-based PGD/PGS in 2013 in Philadelphia [12], several studies have demonstrated the availability and efficiency of using NGS in PGT-A therapy. Fiorentino et al. performed a large preclinical and blind study to validate the accuracy of NGS-based whole chromosome aneuploidy screening [13]. By comparing the embryos obtained with previously established arrayCGH methodology, they confirmed that NGS has a $100 \%$ aneuploidy diagnosis consistency with arrayCGH.

Table 3 Clinical outcomes in NGS-PGT-A group and SNP-PGT-A group

\begin{tabular}{|c|c|c|c|}
\hline Clinical measures & NGS based PGT-A & SNP array based PGT-A & $P$-value \\
\hline No. of couples participating & 805 & 613 & \\
\hline No. of couples with transfer & 522 & 440 & \\
\hline No. of couples without available embryos & 270 & 145 & \\
\hline No. of embryos transferred & 675 & 547 & \\
\hline Transferred cycles & 675 & 547 & \\
\hline Maternal age (mean $\pm S D$ ) of couples with transfer & $29.97 \pm 4.26$ & $29.78 \pm 4.42$ & 0.489 \\
\hline \multicolumn{4}{|l|}{ PGT-A indications } \\
\hline Robertsonian translocation & $21.3 \%(111 / 522)$ & $19.5 \%(86 / 440)$ & 0.510 \\
\hline Reciprocal translocation & $44.6 \%(233 / 522)$ & $53.4 \%(235 / 440)$ & $0.007^{*}$ \\
\hline Inversion & $6.1 \%(32 / 522)$ & $2.5 \%(11 / 440)$ & $0.007^{*}$ \\
\hline Sex chromosome abnormality & $13.8 \%(72 / 522)$ & $8.6 \%(38 / 440)$ & $0.012^{*}$ \\
\hline AMA & $5.0 \%(26 / 522)$ & $4.5 \%(20 / 440)$ & 0.753 \\
\hline RM & $7.5 \%(39 / 522)$ & $9.5 \%(42 / 440)$ & 0.248 \\
\hline RIF & $1.7 \%(9 / 522)$ & $1.8 \%(8 / 440)$ & 0.912 \\
\hline Maternal BMI (mean \pm SD) of couples with transfer & $22.76 \pm 3.00$ & $22.70 \pm 2.96$ & 0.799 \\
\hline Basal FSH level (mean +SD) of women with transfer & $6.51 \pm 1.88$ & $6.71 \pm 1.80$ & 0.113 \\
\hline Endometrial thickness (mean + SD) ${ }^{a}$ & $9.8 \pm 1.91$ & $9.7 \pm 1.94$ & 0.544 \\
\hline Clinical pregnancy rate ${ }^{b}$ & $50.5 \%(341 / 675)$ & $41.7 \%(228 / 547)$ & $0.002^{*}$ \\
\hline Miscarriage rate ${ }^{c}$ & $15.5 \%(54 / 341)$ & $22.8 \%(52 / 228)$ & $0.036^{*}$ \\
\hline Healthy baby rate ${ }^{d}$ & $39.6 \%(267 / 675)$ & $31.4 \%(172 / 547)$ & $0.003^{*}$ \\
\hline
\end{tabular}

${ }^{a}$ Endometrial thickness measured at embryos transferred day

${ }^{\mathrm{b}}$ Clinical pregnancy rate $=$ clinical pregnancy cycles /embryo transferred cycles

${ }^{c}$ Miscarriage rate $=$ embryo lost cycles /clinical pregnancy cycles

${ }^{d}$ Healthy baby rate $=$ healthy babies/ number of transferred embryos

${ }^{*} p<0.05$, statistical significance 
In addition to aneuploidy screening, Sachdeva et al. reported the ability of NGS to detect segmental changes (as small as $14 \mathrm{Mb}$ in size) [20], suggesting it could also be applied in the diagnosis of partial aneuploidy. Furthermore, by comparing NGS results with two other independent methodologies, namely qPCR-based comprehensive chromosome screening and Taqman allelic discrimination assays, Treff et al. validated the use of NGS in PGD treatment for patients with monogenic disease [21].

The current study has not only validated NGS as a reliable technique in selecting chromosomally normal/euploid embryos for transfer, but has also provided evidence of better clinical outcomes for women using NGS-based PGT-A than for those using SNP-based PGT-A. This could be partially explained by the difference in accuracy in detecting chromosomal abnormalities between these two techniques. Tan et al. compared the accuracy by testing 150 blastocysts using both NGS and SNP array [9]. Their results showed that all the tests of normal/balanced blastocysts in the NGS group produced results consistent with those using SNP array, but when it came to chromosomally abnormal blastocysts, seven cases were found to be inconsistent. Further validation was sought by testing the blastocysts using qPCR. This produced results consistent with the NGS tests, indicating that NGS has a higher resolution for chromosomal abnormality detection.

Furthermore, Yin et al. examined 38 blastocysts using NGS and compared the results with those results obtained using SNP array [22]. They demonstrated that both NGS and SNP array could detect embryo aneuploidy with $100 \%$ consistency, but NGS provided higher accuracy in some areas for embryos with unbalanced chromosomal rearrangement [22], likely due to its ability to correct the WGA bias during data analysis. Therefore, the lower accuracy and resolution of SNP array in detecting chromosomally abnormal embryos may result in incorrectly categorizing chromosomally abnormal embryos as being chromosomally normal, leading to their transfer with chromosomal abnormal embryos, and resulting in a miscarriage.

In addition, recent studies have shown that the use of NGS to detect mosaicism can be carried out to a much greater sensitivity than SNP array [23, 24]. By using SNP array, if ideal results are obtained, mosaicism associated with proportions of aneuploid cells ranging from 40 to $60 \%$ could be detected with a high degree of confidence [23]. Proportions of abnormal cells outside this range are indistinguishable between either normality or nonmosaic aneuploidy. In contrast, using NGS, mosaicism could be detected in proportions of abnormal cells ranging from 20 to $80 \%$ [25]. Meanwhile, mosaic embryo transfer results in a low implantation rate, pregnancy rate and a higher miscarriage rate compared with chromosomal normal embryo transferring [26]. Thus, the transfer of undetectable mosaic embryos which were falsely identified as normal embryos by SNP array may partly explain the worse clinical outcomes observed in MDA-SNP-PGT-A.

Different WGA methods used in the current study can also be an potential reasons for the different clinical outcomes (MDA for SNP-PGT-A and MALBAC for NGSPGT-A). MALBAC was found to have a higher genomic coverage, level of specificity, uniformity and reproducibility than MDA in single cell sequencing [27]. However, low-coverage sequencing data reads, as few as 0.1 million, were shown to be able to identify all types of aneuploidy accurately [28]. In addition, recent study found that MALBAC had a higher success rates in detecting all the copy number variants compared with MDA at the single-cell level. While, when five or more cells were used as template, these two methods did not differ significantly [29]. Considering five to ten biopsy cells were used in this study, we hypothesis the effects of different WGA methods on clinical outcomes may not be the main cause of different clinical outcomes observed between MDA-SNP-PGT-A and MALBAC-NGS-PGT-A groups. However, one major limitation of the current study might be that our study cohort was retrospectively established, leaving potential confounding factors unestimated. A prospective, multi-center randomly controlled study is in need to increase the solidity of our conclusions. Additionally, there are 29 couples still waiting for embryo transfer and 15 couples with ongoing pregnancy, we are unable to obtain the complete pregnancy outcomes and healthy baby rate. This represents a minor limitation of this study, but the relatively large study cohort might alleviate the effects of this limitation exerted on our research conclusions.

Based on the better clinical outcomes indicated in this study, and the continuously decreasing cost of NGS, it is anticipated that NGS will play an important role in PGT-A therapy. The current study is the largest one to date in evaluating MALBAC-NGS-PGT-A clinical outcomes. Our subsequent efforts will be directed at testing the difference in diagnostic accuracy between NGS and SNP array in detecting chromosomal abnormalities in biopsied embryos.

\section{Conclusion}

This is the largest study reporting the extensive application of NGS-based PGT-A and comparing the clinical outcomes of MALBAC-NGS-PGT-A and MDA-SNPPGT-A. The results provide further evidence supporting the wider use of NGS in PGT-A, not only for its lower cost but also for its better clinical outcomes. 


\section{Abbreviations}

ART: Assisted reproductive technology; PGT-A: Preimplantation genetic testing for aneuploidy; FISH: Fluorescence in situ hybridization; SNP: Single nucleotide polymorphism; NGS: Next generation sequencing; WGA: Whole genome amplification; MDA: Multiple displacement amplification; MALBAC: Multiple annealing and looping-based amplification cycles; COS: Controlled ovarian stimulation; GnRH-a: Gonadotropin releasing hormone agonist; FSH: Follicle stimulating hormone; BMI: Body mass index; UPD: Uniparental disomy

\section{Acknowledgements}

The authors sincerely acknowledge the staff of Center for Reproductive Medicine, The First Affiliated Hospital of Zhengzhou University for their diligent work, and all the patients for their selfless participation.

\section{Authors' contributions}

YS and WN designed the study. YS,WN and LW proposed the manuscript. WN, JX, LW and YL performed the experiments. HJ, WS and GL collected the samples. FW and HS analyzed the data. All authors have critically reviewed the manuscript and approved of the final version as submitted.

\section{Funding}

The work was supported by National Natural Science Foundation of China (81701443 to Wenbin Niu and 81820108016 to Yingpu Sun), National Key R\&D Program of China (2019YFA0110900 to Yingpu Sun),Clinical Medical Research Fund of Chinese Medical Association-Reproductive Medicine Research and Development Projects for Youth Grant (17020250694 to Wenbin Niu and 17020210690 to Fang Wang), Youth Innovation Project of the First Affiliated Hospital of Zhengzhou University (to Wenbin Niu) and Science and Technology Research Project of Henan Education Department (182102310139 to Fang Wang).

\section{Availability of data and materials}

The data that support the study are available upon reasonable request to the corresponding author.

\section{Ethics approval and consent to participate}

This study was approved by the Ethics Committee of The First Affiliated Hospital of Zhengzhou University. All participants provided written informed consent.

\section{Consent for publication}

Not applicable.

\section{Competing interests}

The authors declare that they have no competing interests.

Received: 22 September 2019 Accepted: 30 June 2020

Published online: 03 July 2020

\section{References}

1. Chard T. Frequency of implantation and early pregnancy loss in natural cycles. Baillieres Clin Obstet Gynaecol. 1991;5(1):179-89.

2. Larsen EC, Christiansen OB, Kolte AM, Macklon N. New insights into mechanisms behind miscarriage. BMC Med. 2013;11:154.

3. Lukaszuk K, Pukszta S, Wells D, Cybulska C, Liss J, Plociennik L, Kuczynski W, Zabielska J. Routine use of next-generation sequencing for preimplantation genetic diagnosis of blastomeres obtained from embryos on day 3 in fresh in vitro fertilization cycles. Fertil Steril. 2015;103(4):1031-6.

4. Philipp T, Philipp K, Reiner A, Beer F, Kalousek DK. Embryoscopic and cytogenetic analysis of 233 missed abortions: factors involved in the pathogenesis of developmental defects of early failed pregnancies. Hum Reprod. 2003;18(8):1724-32.

5. Vanneste $E$, Voet T, Le Caignec C, Ampe M, Konings P, Melotte C, Debrock S, Amyere M, Vikkula M, Schuit F, et al. Chromosome instability is common in human cleavage-stage embryos. Nat Med. 2009;15(5):577-83.

6. Schoolcraft WB, Katz-Jaffe MG. Comprehensive chromosome screening of trophectoderm with vitrification facilitates elective single-embryo transfer for infertile women with advanced maternal age. Fertil Steril. 2013;100(3):615-9.
7. Schoolcraft WB, Fragouli E, Stevens J, Munne S, Katz-Jaffe MG, Wells D. Clinical application of comprehensive chromosomal screening at the blastocyst stage. Fertil Steril. 2010;94(5):1700-6.

8. Harper J, Geraedts J, Borry P, Cornel MC, Dondorp WJ, Gianaroli L, Harton G, Milachich T, Kaariainen $\mathrm{H}$, Liebaers I, et al. Current issues in medically assisted reproduction and genetics in Europe: research, clinical practice, ethics, legal issues and policy. Hum Reprod. 2014;29(8):1603-9.

9. Tan Y, Yin X, Zhang S, Jiang H, Tan K, Li J, Xiong B, Gong F, Zhang C, Pan X, et al. Clinical outcome of preimplantation genetic diagnosis and screening using next generation sequencing. Gigascience. 2014;3(1):30.

10. Treff NR, Su J, Tao X, Levy B, Scott RT Jr. Accurate single cell 24 chromosome aneuploidy screening using whole genome amplification and single nucleotide polymorphism microarrays. Fertil Steril. 2010;94(6):2017-21.

11. Tan YQ, Tan K, Zhang SP, Gong F, Cheng DH, Xiong B, Lu CF, Tang XC, Luo $\mathrm{KL}$, Lin $\mathrm{G}$, et al. Single-nucleotide polymorphism microarray-based preimplantation genetic diagnosis is likely to improve the clinical outcome for translocation carriers. Hum Reprod. 2013;28(9):2581-92.

12. Aleksandrova N, Shubina E, Ekimov A, Kodyleva T, Mukosey I, Makarova N, Kulakova E, Levkov L, Trofimov D, Sukhikh G. Comparison of the results of preimplantation genetic screening obtained by a-CGH and NGS methods from the same embryos. Gynecol Endocrinol. 2016;32(sup2):1-4.

13. Fiorentino F, Biricik A, Bono S, Spizzichino L, Cotroneo E, Cottone G, Kokocinski F, Michel CE. Development and validation of a next-generation sequencing-based protocol for 24-chromosome aneuploidy screening of embryos. Fertil Steril. 2014;101(5):1375-82.

14. Zhang W, Liu Y, Wang L, Wang H, Ma M, Xu M, Xu X, Gao Z, Duan J, Cram $D S$, et al. Clinical application of next-generation sequencing in preimplantation genetic diagnosis cycles for Robertsonian and reciprocal translocations. J Assist Reprod Genet. 2016;33(7):899-906.

15. Hou Y, Wu K, Shi X, Li F, Song L, Wu H, Dean M, Li G, Tsang S, Jiang R, et al. Comparison of variations detection between whole-genome amplification methods used in single-cell resequencing. Gigascience. 2015;4:37.

16. Bolger AM, Lohse M, Usadel B. Trimmomatic: a flexible trimmer for Illumina sequence data. Bioinformatics. 2014;30(15):2114-20.

17. Li H, Durbin R. Fast and accurate short read alignment with burrowswheeler transform. Bioinformatics. 2009;25(14):1754-60.

18. Zhang C, Zhang C, Chen S, Yin X, Pan X, Lin G, Tan Y, Tan K, Xu Z, Hu P, et al. A single cell level based method for copy number variation analysis by low coverage massively parallel sequencing. PLoS One. 2013;8(1):e54236.

19. Xu J, Niu W, Peng Z, Bao X, Zhang M, Wang L, Du L, Zhang N, Sun Y. Comparative study of single-nucleotide polymorphism array and next generation sequencing based strategies on triploid identification in preimplantation genetic diagnosis and screen. Oncotarget. 2016;7(49):81839-48.

20. Sachdeva K, Discutido R, Albuz F, Almekosh R, Peramo B. Validation of nextgeneration sequencer for 24-chromosome aneuploidy screening in human embryos. Genet Test Mol Biomarkers. 2017;21(11):674-80.

21. Treff NR, Fedick A, Tao X, Devkota B, Taylor D, Scott RT Jr. Evaluation of targeted next-generation sequencing-based preimplantation genetic diagnosis of monogenic disease. Fertil Steril. 2013;99(5):1377-84 e1376.

22. Yin X, Tan K, Vajta G, Jiang H, Tan Y, Zhang C, Chen F, Chen S, Zhang C, Pan $X$, et al. Massively parallel sequencing for chromosomal abnormality testing in trophectoderm cells of human blastocysts. Biol Reprod. 2013;88(3):69.

23. Munne $S$, Wells $D$. Detection of mosaicism at blastocyst stage with the use of high-resolution next-generation sequencing. Fertil Steril. 2017;107(5): 1085-91.

24. Friedenthal J, Maxwell SM, Munne S, Kramer Y, McCulloh DH, McCaffrey C, Grifo JA. Next generation sequencing for preimplantation genetic screening improves pregnancy outcomes compared with array comparative genomic hybridization in single thawed euploid embryo transfer cycles. Fertil Steril. 2018;109(4):627-32.

25. Lai HH, Chuang TH, Wong LK, Lee MJ, Hsieh CL, Wang HL, Chen SU. Identification of mosaic and segmental aneuploidies by next-generation sequencing in preimplantation genetic screening can improve clinical outcomes compared to array-comparative genomic hybridization. Mol Cytogenet. 2017;10:14

26. Fragouli E, Alfarawati S, Spath K, Babariya D, Tarozzi N, Borini A, Wells D. Analysis of implantation and ongoing pregnancy rates following the transfer of mosaic diploid-aneuploid blastocysts. Hum Genet. 2017;136(7):805-19.

27. Lu L, Lv B, Huang K, Xue Z, Zhu X, Fan G. Recent advances in preimplantation genetic diagnosis and screening. J Assist Reprod Genet. 2016;33(9):1129-34. 
28. Pasaniuc B, Rohland N, McLaren PJ, Garimella K, Zaitlen N, Li H, Gupta N, Neale BM, Daly MJ, Sklar P, et al. Extremely low-coverage sequencing and imputation increases power for genome-wide association studies. Nat Genet. 2012:44(6):631-5.

29. Liu W, Zhang H, Hu D, Lu S, Sun X. The performance of MALBAC and MDA methods in the identification of concurrent mutations and aneuploidy screening to diagnose beta-thalassaemia disorders at the single- and multiple-cell levels. J Clin Lab Anal. 2018;32(2):e22267.

\section{Publisher's Note}

Springer Nature remains neutral with regard to jurisdictional claims in published maps and institutional affiliations.

Ready to submit your research? Choose BMC and benefit from:

- fast, convenient online submission

- thorough peer review by experienced researchers in your field

- rapid publication on acceptance

- support for research data, including large and complex data types

- gold Open Access which fosters wider collaboration and increased citations

- maximum visibility for your research: over $100 \mathrm{M}$ website views per year

At BMC, research is always in progress.

Learn more biomedcentral.com/submissions 